\title{
Genetic Alterations Between Primary Head and Neck Squamous Cell Carcinoma and Recurrence After Radiotherapy
}

\author{
Recurrence, Genetically Related Cancer, or Second Primary? \\ Alberto Deganello, MD'; Alessandro Franchi, MD²; lacopo Sardi, MD³; Lorenzo Pignataro, MD $^{4}$; \\ C. René Leemans, MD, $\mathrm{PhD}^{5}$; and Oreste Gallo, $\mathrm{MD}^{1}$
}

\begin{abstract}
BACKGROUND: In the attempt to characterize the genetic bases of recurrent head and neck squamous cell carcinoma (HNSCC) after radiotherapy (RT), the authors compared the molecular profiles of primary tumors and recurrences. METHODS: TP53 gene status and instability at 10 microsatellite markers were determined in pre-RT lesions and corresponding local recurrences in a series of 16 HNSCCs. RESULTS: Eight (50\%) HNSCCs showed both TP53 and microsatellite instability (MSI) status concordance in pre- and postirradiation biopsies; 3 (18.7\%) showed discordance of both TP53 and MSI status; and finally 5 (31.2\%) had discordance at only 1 genetic test. Accordingly, the authors interpreted as true recurrence the 8 concordant cases, and as true second primary malignancies the 3 discordant ones. In the remaining 5 cases with partial DNA correspondence, the exact nature of the new lesion only partially related to the original cancer is a matter of discussion. Patients showing the same mutations among pre- and post-RT HNSCCs had a longer disease-free interval (DFI) and better survival than those showing discordant genetic features (log-rank test, $P=.0045$ ). CONCLUSIONS: Post-RT recurrent HNSCCs are genetically heterogeneous. The genetic characterization of the recurrence, especially in those cases with a particularly short DFI showing partially discordant mutations, might have a useful clinical relevance in the restaging process. Cancer 2010;00:000-000. (c) 2010 American Cancer Society.
\end{abstract}

KEYWORDS: head and neck squamous cell carcinoma, radiotherapy, local recurrence, TP53, microsatellite instability, second field tumor, minimal residual disease.

Radiotherapy (RT) plays a significant role in the treatment of head and neck squamous cell carcinoma (HNSCC). Some tumors fail to respond to this approach because of the appearance of resistant populations. The persistence or recurrence of the disease after irradiation is usually a poor prognostic factor, because it is frequently associated with unresponsiveness to other therapies and high risk of regional and distant metastases.

Currently, no universally accepted definition of post-RT recurrence HNSCC exists. Furthermore, the time of tumor relapse is also extremely variable, ranging from few months to 5 years post-RT. This heterogeneity may reflect the possibility that the reappearance of a HNSCC in the same field as a previously RT-treated lesion could represent the development of a second primary malignancy in a field that had been exposed to chemical carcinogens, such as tobacco, alcohol, and previous RT.

Recent studies in the area of cancer genetics have better characterized the possibility that, according to Slaughter's hypothesis, unexpected local recurrences after surgery occurring very close to the site of primary lesions (within $2 \mathrm{~cm}$ ) and

\footnotetext{
Corresponding author: Alberto Deganello, MD, Department of Otolaryngology Head and Neck Surgery, University of Florence, V. le Morgagni 85, 50134 Firenze, Italy; Fax: (011) 39055435649; adeganello@hotmail.com

${ }^{1}$ Department of Otolaryngology/Head and Neck Surgery, University of Florence, Florence, Italy; ${ }^{2}$ Department of Human Pathology and Oncology, University of Florence, Florence, Italy; ${ }^{3}$ Department of Oncohematology, University of Florence, A. Meyer Children's Hospital, Florence, Italy; ${ }^{4}$ Department of Otolaryngology of the "Mangiagalli and Regina Elena" Hospital (IRCCS), University of Milan, Milan, Italy; ${ }^{5}$ Department of Otolaryngology/Head and Neck Surgery, VU University Medical Center, Amsterdam, The Netherlands
}

The first 2 authors equally contributed to this article.

The authors thank Jacopo Scala, MD, for his help with the layout of figures and tables.

DOI: 10.1002/cncr.24854, Received: March 1, 2009; Revised: June 9, 2009; Accepted: July 2, 2009, Published online in Wiley InterScience (www.interscience.wiley.com) 
with a well-defined time interval from the primary treatment (within 36 months) might be due not only to persistence of cancer cells after treatment, designated as minimal residual disease, but also to the progressive development of a new cancer from tumor-related precursor lesions involving the field. ${ }^{1-4}$ Accordingly, the persistence on surgical margins of genetic alterations clonally related to the primary HNSCC is responsible for a higher risk of true local recurrence..$^{5-7}$ Subsequently, the concept of second field tumor has been introduced for those cases showing a minimal genetic discordance from the primary, such recurrence being considered a new carcinoma clonally related to the primary. ${ }^{8}$ Taken together, this hypothesis has been postulated on the basis of genetic studies on biological samples obtained in surgical fields, where a minimal residual disease or a genetically altered field with progression potential may be left behind by the surgeon, thus being responsible for local relapse.

Conversely, few data exist regarding the pathobiology of the development of local recurrence after primary RT that, at least in theory, might result from different mechanisms from both biological and genetic points of view. Several biological and local factors responsible for radioresistance can create a situation comparable to the persistence of a minimal residual disease in a surgical field. Furthermore, analogously to surgery, irradiation might have an impact only on malignant cells and may not affect preneoplastic cells in the field, which undergo subsequent malignant transformation, thus developing a second primary malignancy genetically unrelated to the original cancer. The transformational effect of irradiation on preneoplastic cells in this latter process remains to be elucidated. In any case, other mechanisms responsible for a local recurrence after RT might exist. In fact, because of heterogeneity in cancer cell subpopulations, ${ }^{9}$ different but genetically related cells might show a different response to irradiation; thus, it is likely that irradiation might exert a selection pressure of more aggressive and radioresistant tumor cells, which could be responsible for persistence/recurrence of the tumor. This persistent/recurring cancer caused by RT selection, therefore, might be genetically related but different from the corresponding preirradiated primary tumor, with potential therapeutic and prognostic implications.

To test this hypothesis and to study the genetic alterations involved in HNSCC post-RT recurrences, we analyzed both TP53 mutations and microsatellite patterns in corresponding specimens of primary tumors and recurrences after RT in HNSCC patients who failed after pri- mary RT. These patients were selected from a larger historical series of 85 consecutive patients treated by primary RT. ${ }^{10}$

\section{MATERIALS AND METHODS \\ Patients}

From a historical series of 85 consecutive patients treated by primary RT in whom TP53 gene analysis in the primary lesion revealed the presence of a mutation, ${ }^{10}$ we selected a group of 16 patients in whom a recurrent HNSCC appeared within 36 months and in a field within $2 \mathrm{~cm}$ from the index tumor. According to site, there were 12 squamous cell carcinomas (SCCs) of the larynx, 3 of the oral cavity, and 1 of the oropharynx.

\section{TP53 Gene Analysis}

Formalin-fixed, paraffin-embedded tissue sections (7-8 $\mu \mathrm{m})$ were used to microdissect tumor tissue from normal tissue, which was then used for DNA extraction. Exons 5-8 of the TP53 gene were amplified using the primers described by Gallo et al. ${ }^{10}$ Amplification consisting of 32 cycles was carried out in $25 \mu \mathrm{L}$ of $1.5 \mathrm{mM} \mathrm{MgCl}_{2}$ Perkin Elmer Cetus (Wellesley, Mass) buffer with $1 \mu \mathrm{M}$ of exonflanking primer set, $50 \mu \mathrm{M}$ each deoxynucleotide triphosphate, and 0.5 U Perkin Elmer Cetus AmpliTaq. Temperature and time for the reaction cycles were $95^{\circ} \mathrm{C}$ for 1 minute, $62^{\circ} \mathrm{C}$ for 1 minute, and $72^{\circ} \mathrm{C}$ for 30 seconds. Polymerase chain reaction (PCR) products were heat denatured and subjected to single-stranded conformational polymorphism (SSCP) analysis using electrophoresis on $6 \%$ polyacrylamide gels with $5 \%$ to $10 \%$ glycerol. Electrophoresis was performed at $45 \mathrm{~W}$ for 4 hours at $4^{\circ} \mathrm{C}$; the gels were silver stained and dried on filter paper. All samples were subjected to PCR and SSCP analysis at least twice to confirm the results obtained. To exclude the silent CGA/CGG dimorphism in codon 213, PCR products from samples showing SSCP abnormalities in exon 6 were subjected to restriction analysis with TaqI. Loss of heterozygosity $(\mathrm{LOH})$ was evaluated in those samples missing mutation in relapsed tumors after RT. The polymorphism revealed by MspI digestion of a 107-bp fragment obtained from amplification of p53 intron 6 was used. ${ }^{11}$ Another intragenic short tandem repeat polymorphism (AAAAI) $\mathrm{n}$ was investigated. ${ }^{12}$

Nucleotide sequence was performed after elution from acrylamide gels of abnormal bands detected by SSCP analysis and amplification by PCR using the same primers as used for SSCP analysis, with a modified $5^{\prime}$ end to contain the M13(-20) sequence. These PCR products 
Table 1. Characteristics of 10 Microsatellite Markers Used in This Study

\begin{tabular}{|c|c|c|c|c|}
\hline Marker & $\begin{array}{l}\text { Chromosome } \\
\text { Localization }\end{array}$ & Gene & Repeat Motif & Primer Sequences (F/R) \\
\hline D2S123 & $2 \mathrm{p} 16$ & hMSH2 & $\mathrm{CA}$ & $\begin{array}{l}\text { 5'-GTGACTTGGATACCATCTATCTATC-3' } \\
\text { 5'-CTGCCTITAACAGTGCTATTT-3' }\end{array}$ \\
\hline D3S47 & $3 q 21-24$ & RPN1 & $\mathrm{CA}$ & $\begin{array}{l}\text { 5'-CGGGTACATTGGCCTGTAAG-3' } \\
\text { 5'-GAGACTACGGCATTGCCA-3' }\end{array}$ \\
\hline BAT25 & $4 \mathrm{q} 12$ & c-Kit & A & $\begin{array}{l}\text { 5'-TCGCCTCCAAGAATGTAAGT-3' } \\
\text { 5'-TCTCATTTAACTATGGCTC-3' }\end{array}$ \\
\hline IFN- $\alpha$ & $9 p 21$ & p16INK & $\mathrm{CA}$ & $\begin{array}{l}\text { 5'-GTAAGGTGGAAACCCCCACT-3' } \\
\text { 5'-TGCGCGTTAAGTTAATTGGTT-3' }\end{array}$ \\
\hline D9S156 & $9 p 21$ & p16INK & CA & $\begin{array}{l}\text { 5'-ATCACTITAACTGAGGAGG-3' } \\
\text { 5'-AGATGGTGGTGAATAGAGGG-3' }\end{array}$ \\
\hline D9S171 & $9 p 21$ & p16INK & $\mathrm{CA}$ & $\begin{array}{l}\text { 5'-AGCTAAGTGAACCTCATCTCTGTCT-3' } \\
\text { 5'-ACCCTAGCACTGATGGTATAGTCT-3' }\end{array}$ \\
\hline D16S260 & 16q22.1 & E-cadherin & $\mathrm{CA}$ & $\begin{array}{l}\text { 5'-GGTTGAGATGCTGACATGC-3' } \\
\text { 5'-CAGGGTGGCTGTTATAATG-3' }\end{array}$ \\
\hline TP53 & 17p12 & p53 & $\mathrm{CA}$ & $\begin{array}{l}\text { 5'-ACTGCCACTCCTTGCCCCATTC-3' } \\
5^{\prime} \text {-AGGGATACTATTCAGCCCGAGGTG-3' }\end{array}$ \\
\hline D17S261 & 17p12 & p53 & $\mathrm{CA}$ & $\begin{array}{l}5^{\prime} \text {-CAGGTTCTGTCATAGGACTA-3' } \\
5^{\prime} \text {-TTCTGGAAACCTACTCCTGA-3' }\end{array}$ \\
\hline D21S1245 & $21 \mathrm{q} 21$ & AML-1 & AAAG & $\begin{array}{l}\text { 5'-GTCAGTATTACCCTGTTACCA-3' } \\
5^{\prime} \text {-GTTGAGGATTITGCATCAGT-3' }\end{array}$ \\
\hline
\end{tabular}

F indicates forward; $R$, reverse; IFN, interferon.

were purified on $2 \%$ NuSieve gels, phenol extracted, and subjected to automated sequencing with the Taq Dye Primer Cycle Sequencing kit (Applied Biosystems, Foster City, Calif). Both strands were sequenced for confirmation of the mutations. TP53 wild-type samples were directly sequenced from the original PCR products.

\section{Microsatellite Analysis}

Genomic DNA was extracted from peripheral blood and surgical samples as previously described. ${ }^{7}$ Ten microsatellite loci were amplified using published oligonucleotides. Chromosomal location, type of polymorphism, sequences, annealing temperatures, and references are reported in Table 1. To facilitate multiplexing, the primers were designed to give rise to PCR products of a size difference of at least $100 \mathrm{bp}$. The PCR reactions were carried out in a volume of $30 \mu \mathrm{L}$ and included 50 to $100 \mathrm{ng}$ of genomic DNA, 10mM Tris-HCl (pH 8), $50 \mathrm{mM} \mathrm{KCl,} 1.5 \mathrm{mM}$ $\mathrm{MgCl} 2,0.1 \%$ gelatin, $0.2 \%$ formamide, 2 to $8 \mathrm{pmol}$ of each oligonucleotide primer, $200 \mu \mathrm{mol}$ of each deoxynucleotide triphosphate, and $0.5 \mathrm{U}$ of Taq polymerase. After an initial denaturation at $94^{\circ} \mathrm{C}, 40$ cycles at 1 minute each were performed at the annealing temperature $\left(52-60^{\circ} \mathrm{C}\right.$, dependently by primers) and at $72^{\circ} \mathrm{C}$. A final extension was done at $72^{\circ} \mathrm{C}$ for 7 minutes.
Amplified PCR fragments were analyzed by $8 \%$ to $12 \%$ polyacrylamide gel electrophoresis and run at $20 \mathrm{~W}$ for 3 to 4 hours. Bands were detected by silver staining as already described. ${ }^{13}$ Microsatellite instability was scored when the PCR products from the tumor shifted up or down in respect to the band pattern of normal tissues, or when there was evidence of $\mathrm{LOH}$, defined as a $>50 \%$ visual reduction in the signal intensity of 1 allele. In the case of borderline allelic losses, the PCR reactions were repeated and quantified by densitometric analysis (GS670 Imaging Densitometer, Bio-Rad Laboratories, Hercules, Calif). In instances where the normal and tumor DNAs did not amplify equally, the signals from the photographic bands from the allele retained by the tumor were normalized to those of the corresponding bands of the control tissue either by repeat electrophoresis of adjusted volumes of PCR products or by quantitative densitometry.

\section{Statistical Analysis}

Statistical analysis was performed using Stata software (Stata Corporation, College Station, Tex). Comparison of TP53 gene status and pattern of $\mathrm{LOH} / \mathrm{microsatellite}$ instability (MSI) between the index tumor and the recurrence where analyzed for their impact on disease-free interval (DFI) between RT treatment and detection of the recurrence and for actuarial survival after salvage surgery 
using the Kaplan-Meier method and then using the logrank test for comparison between 2 groups.

\section{RESULTS}

\section{Comparison of TP53 Gene Status in} Pre- and Postirradiation Tumor Samples

According to the site of mutation, we observed a TP53 point mutation in 4 cases in exon 5 (all were laryngeal SCCs), 1 case in exon 6 (a laryngeal SCC), 5 cases in exon 7 (3 were laryngeal SCCs), and finally 5 cases in exon 8 (3 were laryngeal SCCs). Moreover 1 laryngeal carcinoma showed a band shift in both exon 5 and exon 8 .

An identical TP53 gene mutation was detected in both pre- and postirradiated failed tumors in 11 of 16 (68.75\%) cases analyzed. Exon 5 and 8 were prevalently affected in this group.

Conversely, 5 tumors showed a different TP53 gene status in comparison with the original preirradiated primary tumors. In detail, all 5 (31.25\%) cases lost their original TP53 mutations in the postirradiated biopsy specimens; the missed mutations clustered in exon 5 in 4 cases, whereas 1 case involved exon 8 .

To assess the nature of the lack of TP53 mutations in the samples from relapsed tumors that were originally mutated, preirradiated tumor samples were typed at 2 intragenic $\mathrm{p} 53$ polymorphisms. ${ }^{10,11}$ All 5 cases were informative for at least 1 system and were assayed for $\mathrm{LOH}$ in the postirradiated samples; no allelic losses were found.

\section{Comparison of LOH/MSI Patterns in Pre- and Postirradiation Tumor Samples}

Microsatellite analysis was performed in pre-RT and postRT tumor biopsies from the 16 TP53 mutated informative cases. In 8 of 16 patients, different LOH/MSI patterns were documented in pre-RT and post-RT recurring SCC. In total, 7 discordant allelic losses and 8 discordant microsatellite alterations were found in the LOH/MSI genetic assessment (Fig. 1). In 2 cases, Patients 2 and 11, 2 discordant losses and 1 microsatellite alteration were documented comparing LOH/MSI pattern from pre-RT and post-RT samples. In 3 cases (Patients 4, 12, and 16), 1 discordant allelic loss and 1 microsatellite alteration were noted between pre- and post-RT biopsies. Finally, Patients 1,8 and 10 showed a single genetic discordance as a microsatellite alteration between pre- and post-RT tumors.

Considering the comparison between pre- and postRT SCC, we documented the appearance of new genetic alterations in post-RT tumor biopsies from all 3 cases with complete discordance of both TP53 and LOH/MSI status, whereas in the 3 cases with TP53 concordance and LOH/MSI discordance, the postirradiated sample showed lack of genetic alteration at microsatellite loci.

\section{Cumulative Comparison of TP53 Gene Status and Pattern of $\mathrm{LOH} / \mathrm{MSI}$}

Overall, the comparison of genetic assessment including TP53 gene and microsatellite analyses showed that in 8 cases (Patients 3, 5, 6, 7, 9, 13, 14, and 15) a complete genetic concordance was documented, whereas in the remaining 8 cases there was discordance (Fig. 2). In detail, in 3 cases (Patients 2, 4, and 12) both TP53 gene status and LOH/MSI pattern were discordant; in another 3 cases (Patients 1, 8, and 16) despite a TP53 concordance between pre- and postirradiation tumor samples, a different LOH/MSI pattern was documented, and finally in the remaining 2 cases (Patients 10 and 11) a genetic concordance was documented only in the LOH/MSI pattern, whereas the post-RT samples lost the TP53 gene mutation documented in the corresponding preirradiation tumor biopsy.

\section{Clinical Outcome According to Genetic Analysis}

Follow-up data were available on all informative patients and revealed differences in both disease-free and overall survival. We found that those patients without changes in TP53 gene status and LOH/MSI pattern between preand post-RT had a longer DFI before relapse and better survival when compared with those showing a discordant genetic pattern (log-rank test, $P=.0045)$ (Fig. 3). The shortest DFI was documented in those HNSCCs with discordance at 1 genetic test between pre- and postirradiation biopsies (TP53 concordance and LOH/MSI discordance in 3 cases; TP53 discordance and LOH/MSI concordance in 2 cases), and the comparison among the group was still significant (log-rank test, $P=.0012)$.

A surgical salvage after RT failure was attempted for all relapsed cases; however, in 5 patients this was unsuccessful, and patients died from disease. According to genetic assessment, 2 of these (Patients 3 and 15) showed an identical TP53 and LOH/MSI pattern among pre- and postirradiation samples (suggestive for a true recurrence after RT failure), whereas the remaining deaths were documented among 1 patient (Patient 2) with complete discordant genetic pattern (suggestive for a second primary malignancy) and the only 2 patients (Patients 10 and 11) who lost TP53 mutation in recurring lesions 

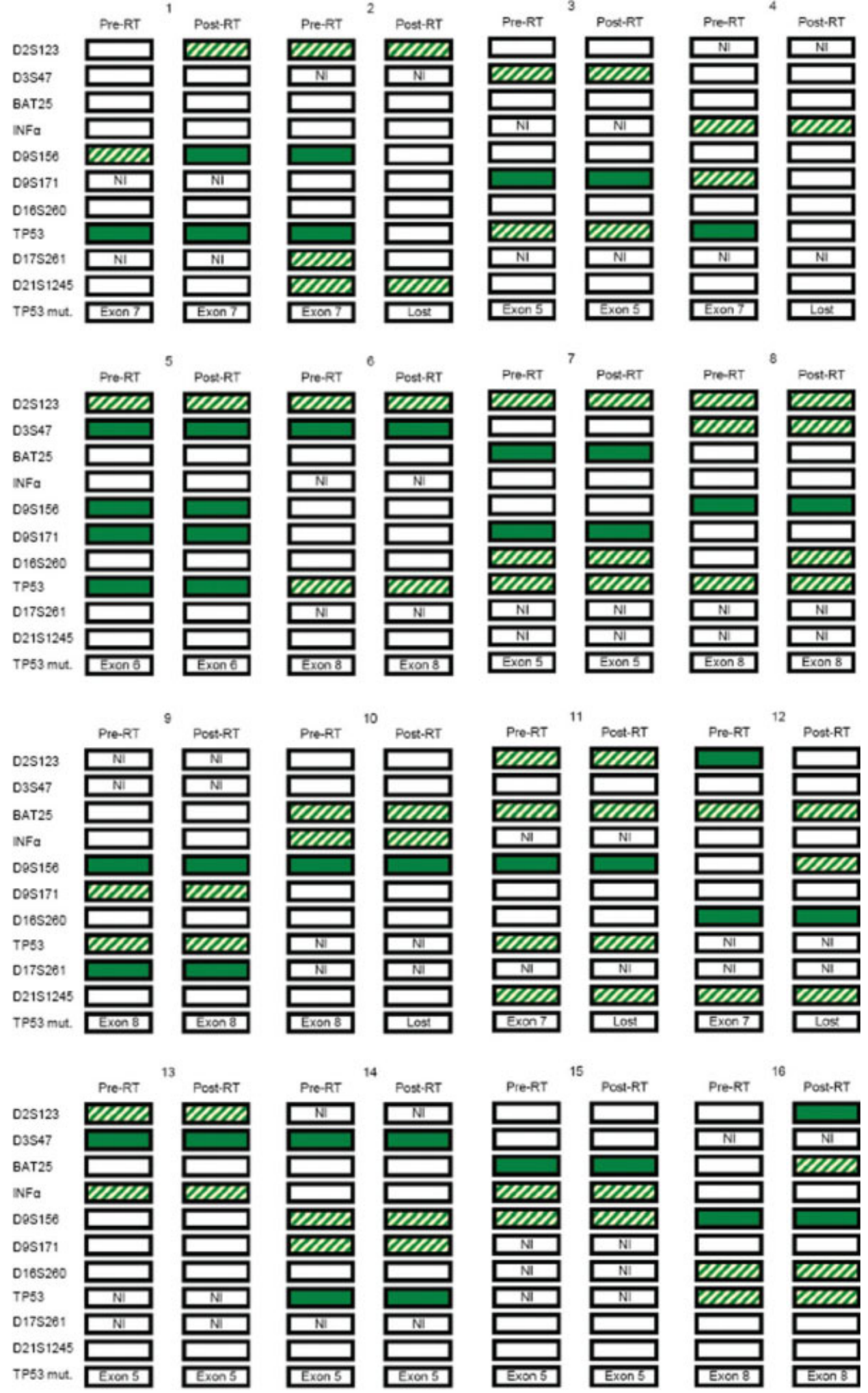

Figure 1. Microsatellite and p53 analysis before (pre) and after (post) radiotherapy (RT) is shown. 


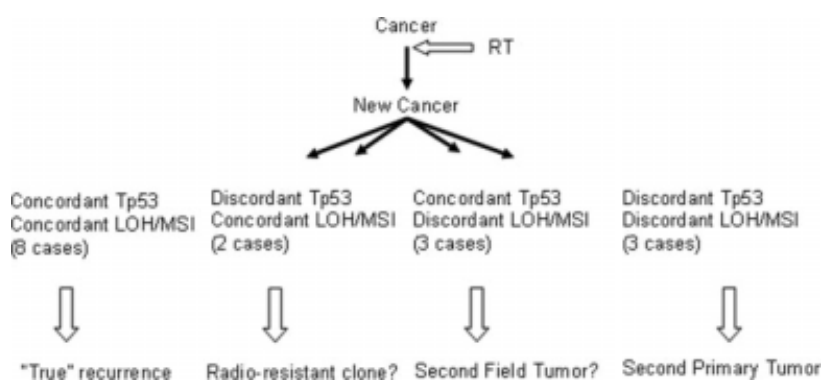

Figure 2. Comparison of genetic assessment is shown. RT indicates radiotherapy; LOH, loss of heterozygosity; MSI, microsatellite instability.

without any discordance in LOH/MSI pattern analyzed. According to Kaplan-Meier survival analysis, differences among groups were statistically significant (log-rank test, $P=.0002)$.

\section{DISCUSSION}

In this study, we attempted to define the pathobiology of locally recurring HNSCC after curative RT. In this light, we selected patients who had relapse within a period of 36 months after the end of radiation treatment and at a distance $<2 \mathrm{~cm}$ from the first carcinoma, and we compared TP53 gene status and microsatellite analyses in samples of pre- and post-RT recurring lesions. Here, we first postulate that a local recurrence after primary radiation therapy might have similar aspects but also a different pathobiology from a recurrence after surgery.

In our limited series, we found 8 cases with complete concordance of the genetic tests that could be interpreted as true RT-failed recurrences of the original HNSCC. Furthermore, we detected a complete discordance between pre- and post-RT biopsies in 3 cases, indicating the appearance of a new carcinoma genetically unrelated to the primary tumor and, thus, definable as a second primary.

Interestingly, in the remaining 5 cases, genetic analyses of both TP53 and LOH/MSI status showed pre- and post-RT concordance in only 1 test.

In this series, we observed a better disease-free survival in patients with discordance of both TP53 gene and LOH/MSI status (second primary) in comparison with the cases showing genetic discordance at 1 test, and among them we documented a shorter DFI and overall survival in the 2 cases with lack of TP53 mutation in the postirradiation sample. The absence of new additional genetic alterations with the exception of the

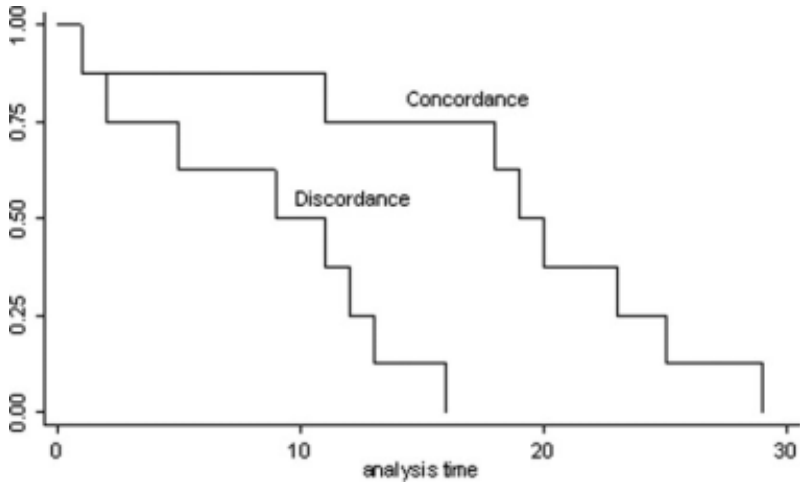

Figure 3. Kaplan-Meyer disease-free interval (radiotherapy recurrence) is shown between patients with concordance and those with discordance of TP53 and loss of heterozygosity/ microsatellite instability mutations.

lack of TP53 mutation in these 2 cases, together with their worse prognosis, could suggest that these relapses reflect a RT-induced selection of more aggressive, less RT-sensitive clones within the tumor mass. In other words, these 2 cases might represent genetically selected true recurrences. In fact, the particularly short DFI between RT and recurrence ( $<3$ months) clinically classifies them as persistence that should be expected to be totally genetically concordant with the original cancer; the evidence of a genetic discordance might be related to the selection impact of RT confirmed by the worse prognostic impact.

The remaining 3 discordant cases with more favorable prognosis, in which the discordance was limited to LOH/MSI pattern, might represent a new genetically related carcinoma from a common field, second field tumor, in which the role of irradiation in determining its development is not yet defined, but possibly preneoplastic clones genetically related to the primary tumor that are not killed by RT because they are not yet neoplastic subsequently undergo neoplastic transformation.

The possibility of defining the pathobiology of postRT recurring cancers in HNSCC patients also by genetic assessment of tumor DNA represents a challenge for researchers. However, except in the case of detection of solid tumors with a specific histology (ie, sarcoma) or carcinomas with specific RT-induced DNA aberrations suggestive of RT-induced second primary HNSCC ${ }^{14,15}$ the time interval between the development of the primary and of the recurrent cancer might help in most cases. It is likely that a few cancer cells surviving irradiation take less time 
to grow into a clinically detectable tumor (presumably also because of their increased aggressiveness) than a field of preneoplastic cells needs for malignant progression to invasive carcinoma.

In conclusion, we have found that even if the reappearance of a new carcinoma does meet clinical criteria to be considered a recurrence, from the genetic point of view things are more heterogeneous. Whereas a true recurrence or a true second primary HNSCC seem to be clearly definable, less is known about the pathobiology of those carcinomas showing genetic discordance among pre- and post-RT sample biopsies.

Hopefully our results will be more consistently clarified with larger numbers and longer follow-up than in this limited series. If our data are confirmed by further studies, then the genetic characterization of the recurrence, especially in those cases with a particularly short DFI, might acquire a useful clinical relevance, while planning salvage therapeutic strategy.

\section{CONFLICT OF INTEREST DISCLOSURES}

The authors made no disclosures.

\section{REFERENCES}

1. Slaughter DP, Southwick HW, Smejkal W. Field cancerization in oral stratified squamous epithelium; clinical implications of multicentric origin. Cancer. 1953;6:963968.

2. Brennan JA, Mao L, Hruban RH, et al. Molecular assessment of histopathological staging in squamous cell carcinoma of the head and neck. N Engl J Med. 1995;332:429435.

3. Sardi I, Franchi A, Ferriero G, et al. Prediction of recurrence by microsatellite analysis in head and neck cancer. Genes Chromsomes Cancer. 2000;29:201-206.
4. van Houten VMM, Leemans CR, Kummer JA, et al. Molecular diagnosis of surgical margins and local recurrence in head and neck cancer patients: a prospective study. Clin Cancer. 2004;10:3614-3620.

5. Braakhuis BJM, Tabor MP, Kummer JA, Leemans CR, Brakenhoff RH. A genetic explanation of Slaughter's concept of field cancerization: evidence and clinical implications. Clin Cancer Res. 2003;63:1727-1730.

6. Partridge M, Li SR, Pateromichelakis S, et al. Detection of minimal residual cancer to investigate why oral tumors recur despite seemingly adequate treatment. Clin Cancer Res. 2000;6:2718-2725.

7. Tabor MP, Brakenhoff RH, Ruijter-Schippers HJ, Kummer JA, Leemans CR, Braakhuis BJM. Genetically altered fields as origin of locally recurrent head and neck cancer: a retrospective study. Clin Cancer Res. 2004;10:3607-3613.

8. Braakhuis BJM, Tabor MP, Leemans CR, Van der Waal I, Snow GB, Brakenhoff RH. Second primary tumors and field cancerization in oral and oropharyngeal cancer; molecular techniques provide new insights and definitions. Head Neck. 2002;24:198-206.

9. Waridel F, Estreicher A, Bron L, et al. Field cancerisation and polyclonal p53 mutation in the upper aerodigestive tract. Oncogene. 1997;14:163-169.

10. Gallo O, Chiarelli I, Bianchi S, Calzolari A, Simonetti L, Porfirio B. Loss of p53 gene mutation after irradiation is associated with increased aggressiveness in recurring head and neck cancer. Clin Cancer Res. 1996;2:15771582 .

11. McDaniel I, Carbone D, Takahashi I, et al. The MspI polymorphism in intron 6 of p53 (1P53) detected by digestion of PCR products. Nucleic Acids Res. 1991;19:4796.

12. Futreal PA, Barrett JC, Wiseman RW. An Alu polymorphism intragenic to the TP53 gene. Nucleic Acids Res. 1991;19:6977.

13. Dockhorn-Dworniczak B, Dworniczak B, Brommelkamp L, Bulles J, Horst J, Bocker WW. Non-isotopic detection of single strand conformation polymorphism (PCR-SSCP): a rapid and sensitive technique in diagnosis of phenylketonuria. Nucleic Acids Res. 1991;19:2500-2557.

14. International Commission on Radiological Protection.Risks associated with ionizing radiations. Ann ICRP. 1991;22:129.

15. Steeves RA. Neoplasms induced by megavoltage radiation in the head and neck region. Cancer. 1981;47:1770-1774. 